Applied Mathematical Sciences, Vol. 7, 2013, no. 24, 1195 - 1197

HIKARI Ltd, www.m-hikari.com

\title{
On Reoptimization of \\ the Shortest Common Superstring Problem
}

\author{
Vladimir Popov \\ Department of Intelligent Systems and Robotics \\ Ural Federal University \\ 620083 Ekaterinburg, Russia \\ Vladimir.Popov@usu.ru
}

\begin{abstract}
In general, a reoptimization gives us a possibility to obtain a solution for a larger instance from a solution for a smaller instance. In this paper, we consider a possibility of usage of a reoptimization to solve the shortest common superstring problem.
\end{abstract}

Keywords: reoptimization, common superstring, Hamming distance

Investigation of different regularities has become essential in modern computer science (see e.g. [1] - [7]). In this paper, we consider the shortest common superstring problem.

The Shortest Common Superstring Problem (SCS):

InstancE: A collection $\mathcal{S}$ of strings over $\Sigma$ and a positive integer $k$. $\mathcal{S}$ ?

Question: Is there a string $S$ such that $|S| \leq k$ and $S$ is a superstring of

The problem SCS is NP-complete [8]. Moreover, SCS is MAX SNP-hard [9]. The following problem was proposed in [10].

The Shortest Common Superstring Reoptimization Problem With Adding a String (SCS+):

INSTANCE: A substring-free collection $\mathcal{S}$ of strings over $\Sigma$, an optimal solution of $\mathrm{SCS}$ for $\mathcal{S}$, and a string $T \notin \mathcal{S}$ such that $\mathcal{S} \cup\{T\}$ is substring-free.

TASK: Find an optimal solution of SCS for $\mathcal{S} \cup\{T\}$.

We can try to use SCS+ for solution of SCS. However, SCS+ is NPhard [10]. We need some efficient algorithm for SCS+. But, there is no clear evidence for usage of an optimal solution of SCS for $\mathcal{S}$ for finding an optimal solution of SCS for $\mathcal{S} \cup\{T\}$. 
Note that if $\mathcal{S}=\left\{a b^{2}, c^{2} a\right\}$, then $c^{2} a b^{2}$ is the shortest common superstring for $\mathcal{S}$. If $T=c a^{2} b$, then $c^{2} a^{2} b^{2}$ is the shortest common superstring for $\mathcal{S} \cup\{T\}$. So, we can try to use an optimal solution of SCS for $\mathcal{S}$ to solve SCS for $\mathcal{S} \cup\{T\}$ under assumption that an optimal solution of SCS for $\mathcal{S}$ is a subsequence of an optimal solution for $\mathcal{S} \cup\{T\}$.

Proposition 1. For any $n>0$, there is a collection $\mathcal{S}$ and strings $S, T$, and $W$ such that $S$ is the shortest common superstring for $\mathcal{S}, W$ is the shortest common superstring for $\mathcal{S} \cup\{T\}, S$ is not a subsequence of $W$, and $|S|>n$.

Proof. It is easy to check that we can consider $\mathcal{S}=\left\{a^{n} b^{n}, c^{n} a^{n}\right\}, S=$ $c^{n} a^{n} b^{n}, T=b^{n} c^{n}, W=a^{n} b^{n} c^{n} a^{n}$.

Therefore, in general case, we can not extend a shortest common superstring for $\mathcal{S}$ to a shortest common superstring for $\mathcal{S} \cup\{T\}$.

Let $D_{H}(X, Y)$ be the Hamming distance between strings $X$ and $Y$.

Proposition 2. For any $n>0$, there is a collection $\mathcal{S}$ and strings $S, T$, and $W$ such that $S$ is a shortest common superstring for $\mathcal{S}, W$ is a shortest common superstring for $\mathcal{S}, W$ is a shortest common superstring for $\mathcal{S} \cup\{T\}$, $|S|=|W|, D_{H}(S, W)=|W|$, and $|S|>n$.

Proof. It is easy to check that we can consider $\mathcal{S}=\left\{a^{n} b^{n}, b^{n} a^{n}\right\}, S=$ $a^{n} b^{n} a^{n}, T=b a^{n} b, W=b^{n} a^{n} b^{n}$.

So, in general case, knowledge of an optimal solution for $\mathcal{S}$ does not provide any significant advantage in finding of an optimal solution for $\mathcal{S} \cup\{T\}$. Therefore, the assumption that an optimal solution of SCS for $\mathcal{S}$ is a subsequence of an optimal solution for $\mathcal{S} \cup\{T\}$ allows us to solve SCS only in some special case. We consider the following version of SCS+.

The Shortest Common Superstring Reoptimization Problem WiTH AdDition (SCS-AD):

INSTANCE: A substring-free collection $\mathcal{S}$ of strings over $\Sigma$, an superstring $S$ of $\mathcal{S}$, a string $T \notin \mathcal{S}$ such that $\mathcal{S} \cup\{T\}$ is substring-free, and a positive integer $k$.

QUESTION: Is there a string $W$ such that $|W| \leq k, W$ is a superstring of $\mathcal{S} \cup\{T\}$, and $S$ is a subsequence of $W$.

Proposition 3. SCS-AD can be solved in polynomial time.

Proof. Let $\mathcal{S}=\left\{S_{1}, \ldots, S_{n}\right\}$. For simplicity, we use $X[i]$ to denote the $i$ th letter in string $X$, and $X[i, j]$ to denote the substring of $X$ consisting of the $i$ th letter through the $j$ th letter.

Let $S_{i}=S[u[i], v[i]]$, for any $1 \leq i \leq n$ and for some $1 \leq u[i]<v[i] \leq|S|$. Since $\mathcal{S}$ is substring-free, it is easy to see that $u[i] \neq u[j]$, for any $1 \leq i<j \leq n$. For any superstring $S$ of $\mathcal{S}$, we can consider a permutation $\pi=(i[1], \ldots, i[n])$ on the set $\{1, \ldots, n\}$ such that $u[i[1]]<\ldots<u[i[n]]$. It is easy to see that if $v[i[j]]+1<u[i[j+1]]$, for some $1 \leq j<n$, then $S[1, v[i[j]]] S[u[i[j+1]],|S|]$ is a superstring of $\mathcal{S}$. Therefore, we can assume that $u[i[j+1]] \leq v[i[j]]+1$. We define overlap $o(X, Y)$ of strings $X$ and $Y$ by $\max \{p \mid X[i]=Y[j], 1 \leq$ 
$j \leq p,|X|-p+1 \leq j \leq|X|\}$. Since $\mathcal{S}$ is substring-free, we can assume that $u[i[j+1]]=v[i[j]]+1-o\left(S_{i[j]}, S_{i[j+1]}\right)$. So, each permutation $\pi$ uniquely defines some superstring of $\mathcal{S}$. Moreover, for any shortest superstring of $\mathcal{S}$ there is a permutation $\pi$ which defines this superstring. Therefore, we can consider a solution of SCS as a permutation. Since any solution of SCS can be represented by some permutation on the set $\{1, \ldots, n\}$, it is easy to see that there are only $n+1$ positions for $T$.

ACKNOWLEDGEMENTS. The work was partially supported by Analytical Departmental Program "Developing the scientific potential of high school" 8.1616.2011.

\section{References}

[1] A. Gorbenko and V. Popov, Multiple Occurrences Shortest Common Superstring Problem, Applied Mathematical Sciences, 6 (2012), 6573-6576.

[2] A. Gorbenko and V. Popov, On the Longest Common Subsequence Problem, Applied Mathematical Sciences, 6 (2012), 5781-5787.

[3] A. Gorbenko and V. Popov, The set of parameterized k-covers problem, Theoretical Computer Science, 423 (2012), 19-24.

[4] V. Yu. Popov, Computational complexity of problems related to DNA sequencing by hybridization, Doklady Mathematics, 72 (2005), 642-644.

[5] V. Popov, The approximate period problem for DNA alphabet, Theoretical Computer Science, 304 (2003), 443-447.

[6] V. Popov, The Approximate Period Problem, IAENG International Journal of Computer Science, 36 (2009), 268-274.

[7] V. Popov, Multiple genome rearrangement by swaps and by element duplications, Theoretical Computer Science, 385 (2007), 115-126.

[8] J. Gallant, D. Maier, and J.A. Storer, On Finding Minimal Length Superstrings, Journal oj Computer and System Sciences, 20 (1980), 50-58.

[9] A. Blum, T. Jiang, M. Li, J. Tromp, and M. Yannakakis, Linear Approximation of Shortest Superstring, Journal of the ACM, 41 (1994), 630-647.

[10] D. Bilò, H.-J. Böckenhauer, D. Komm, R. Královič, T. Mömke, S. Seibert, and A. Zych, Reoptimization of the Shortest Common Superstring Problem, Lecture Notes in Computer Science, 5577 (2009), 78-91.

Received: December 3, 2012 